\title{
Long-term follow-up after cardiac resynchronization therapy-optimization in a real-world setting: A single-center cohort study
}

\author{
Raphael Korach, Peter C. Kahr, Frank Ruschitzka, \\ Jan Steffel, Andreas J. Flammer, Stephan Winnik \\ University Heart Center, Cardiology, University Hospital Zurich, Switzerland
}

\begin{abstract}
Background: Suboptimal device programming is among the reasons for reduced response to cardiac resynchronization therapy (CRT). However, whether systematic optimization is beneficial remains unclear, particularly late after CRT implantation. The aim of this single-center cohort study was to assess the effect of systematic atrioventricular delay (AVD) optimization on echocardiographic and device parameters.

Methods: Patients undergoing CRT optimization at the University Hospital Zurich between March 2011 and January 2013, for whom a follow-up was available, were included. AVD optimization was based on 12-lead electrocardiography (ECG) and echocardiographic left ventricular inflow characteristics. Parameters were assessed at the time of CRT optimization and follow-up, and were compared between patients with AVD optimization (intervention group) and those for whom no AVD optimization was deemed necessary (control group).

Results: Eighty-one patients with a mean age of $64 \pm 11$ years were included in the analysis. In $73 \%$ of patients, AVD was deemed suboptimal and was changed accordingly. After a median follow-up time of 10.4 (IQR 6.2 to 13.2) months, the proportion of patients with sufficient biventricular pacing (>97\% pacing) was greater in the intervention group (78\%) compared to controls (50\%). Furthermore, AVD adaptation was associated with an improvement in interventricular mechanical delay (decrease of $6.6 \pm$ $\pm 26.2 \mathrm{~ms} v \mathrm{~s}$. increase of $4.3 \pm 17.7 \mathrm{~ms}, p=0.034$ ) and intraventricular septal-to-lateral delay (decrease of $0.9 \pm 48.1 \mathrm{~ms} v$ s. increase of $15.9 \pm 15.7 \mathrm{~ms}, p=0.038$ ), as assessed by tissue Doppler imaging. Accordingly, a reduction was observed in mitral regurgitation along with a trend towards reduced left ventricular volumes.

Conclusions: In this "real-world" setting systematic AVD optimization was associated with beneficial effects regarding biventricular pacing and left ventricular remodeling. These data show that AVD optimization may be advantageous in selected CRT patients. (Cardiol J 2021; 28, 5: 728-737)
\end{abstract}

Key words: cardiac resynchronization therapy, atrioventricular delay, biventricular pacing, left ventricular remodeling

\section{Introduction}

Cardiac resynchronization therapy (CRT) is a life-saving treatment in selected patients with symptomatic heart failure and reduced ejection fraction (HFrEF) [1-3]. In patients with persistent symptoms (New York Heart Association [NYHA] II to ambulatory IV) on optimal medical therapy,

Address for correspondence: Andreas Flammer, MD, and Stephan Winnik, MD, PhD, University Heart Center Zurich,

Raemistr. 100, CH-8091 Zurich, Switzerland, tel: +41(0)442554939 or +41(0)442554782,

e-mail: andreas.flammer@usz.ch or stephan.winnik@usz.ch

Received: 12.06.2019 Accepted: 01.01.2020 Early publication date: 9.01.2020

This article is available in open access under Creative Common Attribution-Non-Commercial-No Derivatives 4.0 International (CC BY-NC-ND 4.0) license, allowing to download articles and share them with others as long as they credit the authors and the publisher, but without permission to change them in any way or use them commercially. 
a left ventricular ejection fraction (LVEF) of $35 \%$ or less, and a wide QRS complex (> $130 \mathrm{~ms}$ ), CRT has been shown to prolong life and reduce the risk for recurrent heart failure (HF) hospitalizations [4-8]. However, about one third of patients remain unresponsive to biventricular pacing and do not exhibit improvement in clinical or hemodynamic parameters $[5,6]$. Several factors may account for this unsatisfactory therapeutic response in this relevant proportion of patients. Apart from ineffective synchronization secondary to suboptimal left ventricular (LV) lead placement or extensive scar tissue, indeliberate patient selection remains a major source of error [7, 9]. However, even after correct LV lead placement and in the absence of extensive scar tissue, response to CRT may not be evident. Such therapy failure may be attributed to suboptimal device programming, specifically with regard to the atrioventricular delay (AVD) and interventricular (VV) interval $[10,11]$. Yet, whether systematic AVD optimization is of prognostic benefit, remains unclear. To date, a number of studies suggest an improvement of clinical, echocardiographic and hemodynamic parameters after AVD optimization. However, the number of patients is very low and follow-up times are short [12-15].

At the documented institution, a standard protocol of echocardiography- and 12-lead electrocardiography (ECG)-guided device optimization after CRT implantation was implemented. It was previously demonstrated that a majority of patients undergoing CRT optimization after implantation presented with suboptimal device settings, particularly regarding AVD [16]. The aim of this study was to evaluate the clinical course after AVD optimization and to study whether patients, in whom the AVD was changed, fared better than those in whom the AVD was left unchanged in this real-world setting.

\section{Methods}

All patients with a CRT-device who underwent CRT optimization at the documented device clinic between March 2011 and January 2013 and in whom at least one follow-up including echocardiography was available were included. CRT devices were implanted according to standard protocols at the University Heart Center Zurich. Patients for CRT implantation were selected based on current guideline recommendations [17]. After implantation, a baseline CRT-optimization was performed on a routine basis, patients referred for CRT implantation from elsewhere underwent baseline optimization in cases of explicit referral. Baseline optimization included a comprehensive device optimization protocol with a complete clinical assessment by a HF specialist, a device interrogation, 12-lead ECGs of intrinsic and paced (BiV, RV, LV) rhythms, and a complete echocardiograph exam with optimization of AVD, if deemed necessary [16]. After baseline optimization, follow-up CRT-optimization was performed in cases of non-response or signs of disease progression, i.e. patients were referred for follow-up CRT optimization if there was a decrease or insufficient increase of LVEF after unexplained HF decompensation or in cases of unexplained progressive decline in exercise capacity.

The need for optimization of AVD was based on the degree of QRS fusion on 12-lead ECG and the presence of LV inflow truncation or fusion as assessed by pulsed wave Doppler echocardiography. For detection of electrical fusion, QRS morphology was assessed on 12-lead ECG during intrinsic rhythm, in biventricular stimulated VVI mode (representing "true" biventricular pacing), during right/left ventricular pacing only, and during CRT pacing under current settings. AVD was then programmed for as long as possible without signs of fusion with intrinsic conduction. Optimal LV filling was subsequently determined according to the iterative method $[18,19]$, i.e., AVD was shortened in steps of $20 \mathrm{~ms}$ under parallel assessment of QRS morphology on a 12-lead ECG and mitral inflow on pulsed wave Doppler echocardiography until truncation of the A-wave indicated impairment of LV filling. In a third step, AVD was increased in steps of $10 \mathrm{~ms}$ until an optimal separation of $E$ and A wave occurred. This was considered an optimal atrioventricular coupling.

For the current study, clinical, echocardiographic and device parameters at the time of echocardiography and 12-lead ECG-guided CRT optimization (baseline visit) and at the time of the follow-up visit were analyzed. Parameters were compared between patients, in whom the AVD was changed at baseline ("intervention group") and those, in whom no adaptation of the AVD was made ("control group") (Fig. 1, Suppl. Fig. 1). Reasons for not changing the AVD were either an interval that was deemed optimal as assessed by the method described above, or if a change in AVD would lead to new QRS fusion or truncation of the A wave. 


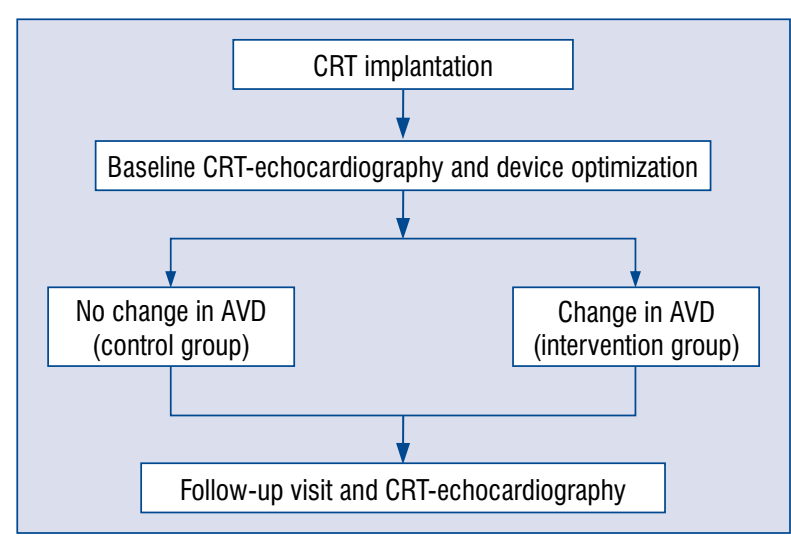

Figure 1. Follow-up flow chart; AVD - atrioventricular delay; CRT - cardiac resynchronization therapy.

\section{Statistical analysis}

Continuous variables are expressed as mean and standard deviation; categorical variables are expressed as proportions. Within-group comparisons (baseline vs. follow-up) were performed using the paired Student t-test for continuous variables and the paired Wilcoxon signed-rank tests for categorical variables. Between-group comparisons (intervention group vs. control group) were done using the unpaired Student t-test and the Mann-Whitney-U-test, where applicable. Distribution of data was assessed by the Shapiro-Wilk test and quantile-quantile (Q-Q) plots. Both data at baseline optimization and at follow-up as well as differences in parameters over time were assessed for normal distribution. Proportions were compared using $\chi^{2}$ tests. Statistical significance was accepted for $\mathrm{p}<0.05$. All p-values are two-sided. Statistical analyses were performed using SPSS version 22 .

\section{Results}

Eighty-one patients undergoing baseline CRT optimization between March 2011 and January 2013 and in whom a consecutive follow-up was available were included in the analysis. Baseline characteristics are summarized in Table 1 . With the exemption of atrial fibrillation (AF), which was absent in the intervention group, and complete atrioventricular block, which was more frequent in the control group, no significant differences were present (Table 1). Median time between CRT implantation and baseline CRT-optimization was 1.7 (IQR 0.4 to 4.2 ) years. Median follow-up time between baseline optimization and follow-up was 10.4 (IQR 6.2 to 13.2) months. Out of 81 patients, 3 patients were hospitalized for HF during follow-up (2 in the intervention group, 1 in the control group). At baseline, 59 (73\%) patients presented with AVD, which was deemed suboptimal either secondary to the presence of QRS fusion on a 12-lead ECG or due to unfavorable LV-filling patterns as assessed by echocardiography [16]. In these patients, AVD was reprogrammed according to the method described above; in the majority of these patients ( $\mathrm{n}=42$, $52 \%$ ) AVD was decreased, secondary to QRS fusion and/or LV inflow fusion. In 17 (21\%) patients AVD was prolonged secondary to $\mathrm{LV}$ inflow truncation. Accordingly, the average AVD was significantly shorter at follow-up compared to baseline (120 \pm $20 \mathrm{~ms}$ at baseline and $100 \pm 29 \mathrm{~ms}$ at follow-up, $\mathrm{p}=0.001) .12(15 \%)$ patients were in AF.

In the overall population, clinical parameters did not change significantly between baseline and the follow-up visit. The proportion of patients with NYHA class II or higher was $79 \%(\mathrm{n}=58 / 73)$ at baseline and $76 \%(\mathrm{n}=57 / 75)$ at follow-up $(\mathrm{p}=0.109)$.

Interestingly, biventricular pacing increased in patients after AVD adjustment over time. While there was no difference in biventricular pacing at baseline, the proportion of patients with a biventricular pacing rate of $>97 \%$ increased significantly by the time of follow-up (78\% in the intervention group vs. $50 \%$ in the control group, $\mathrm{p}=0.021$; Fig. 2). As proof of concept, reassessment of biventricular pacing at follow-up was performed after exclusion of 6 patients with $\mathrm{AF}$ and intact atrioventricular conduction. Biventricular pacing proportions remained significantly higher in the intervention compared to the control group (mean biventricular pacing rate: $94.5 \pm 6.8 \%$ in the control group, $97.5 \pm 4.0 \%$ in the intervention group, $\mathrm{p}=0.022$; percentage of patients with $>97 \%$ biventricular pacing: $44 \%$ in the control group, $78 \%$ in the intervention group, $\mathrm{p}=0.031$; Suppl. Table 1).

Moreover, both interventricular mechanical delay (IVMD) and septal to lateral delay (SLD), as assessed by tissue Doppler imaging, decreased in the intervention group (AVD changed) compared to the control group (AVD unchanged), in which both IVMD and SLD increased from baseline to follow-up (Fig. 3). Although LVEF was not different between the intervention and the control group at follow-up (Fig. 4A), a trend was observed towards reduced end-diastolic LV volumes (Fig. 4B) in the intervention group. Along this line, the proportion of patients with mitral regurgitation, which did not differ between 
Table 1. Parameters at baseline during cardiac resynchronization therapy-optimization.

\begin{tabular}{|c|c|c|c|c|}
\hline Parameters & $\begin{array}{l}\text { Overall population } \\
\quad(\mathrm{n}=81)\end{array}$ & $\begin{array}{l}\text { Control group } \\
(n=22)\end{array}$ & $\begin{array}{l}\text { Intervention group } \\
(\mathrm{n}=59)\end{array}$ & $\mathbf{P}$ \\
\hline Age at implantation (years) & $64 \pm 11$ & $63 \pm 16$ & $64 \pm 9$ & 0.725 \\
\hline Men (n/total) & $63 / 81(78 \%)$ & $17 / 22(77 \%)$ & $46 / 59(78 \%)$ & 0.947 \\
\hline \multicolumn{5}{|l|}{ Co-morbidities } \\
\hline Diabetes mellitus & $20 / 80(25 \%)$ & $7 / 22(31.8 \%)$ & $13 / 58(22.4 \%)$ & 0.386 \\
\hline Hypertension & $44 / 81(54.3 \%)$ & $13 / 22(59.1 \%)$ & $31 / 59(52.5 \%)$ & 0.87 \\
\hline Dyslipidemia & $45 / 81(55.6 \%)$ & $13 / 22(59.1 \%)$ & $32 / 59(54.2 \%)$ & 0.875 \\
\hline Coronary artery disease & $32 / 81(39.5 \%)$ & $8 / 22(36.4 \%)$ & $24 / 59(40.7 \%)$ & 0.724 \\
\hline Atrial fibrillation & $12 / 81(15 \%)$ & $12 / 22(55 \%)$ & $0 / 59(0 \%)$ & $<0.001 *$ \\
\hline \multicolumn{5}{|l|}{ Medication } \\
\hline ACEI/ARBs & $79 / 80(98.8 \%)$ & $21 / 22(96 \%)$ & $58 / 58(100 \%)$ & 0.102 \\
\hline Beta-blockers & $77 / 80(96.3 \%)$ & $21 / 22(96 \%)$ & $56 / 58(96.6 \%)$ & 0.818 \\
\hline Calcium channel blockers & $6 / 80(7.5 \%)$ & $0 / 22(0 \%)$ & $6 / 58(10 \%)$ & 0.117 \\
\hline Spironolactone & $47 / 80(58.8 \%)$ & $12 / 22(55 \%)$ & $35 / 58(60 \%)$ & 0.638 \\
\hline Diuretics & $69 / 80(86.3 \%)$ & $21 / 22(96 \%)$ & $48 / 58(83 \%)$ & 0.141 \\
\hline Digitalis & $10 / 80(12.5 \%)$ & $4 / 22(18.2 \%)$ & $6 / 58(10.3 \%)$ & 0.344 \\
\hline Amiodarone & $12 / 80(15 \%)$ & $4 / 22(18.2 \%)$ & $8 / 58(13.8 \%)$ & 0.624 \\
\hline \multicolumn{5}{|l|}{ Clinical parameters } \\
\hline NYHA class: & & & & 0.946 \\
\hline NYHA I & $15 / 73(20 \%)$ & $5 / 21(24 \%)$ & $10 / 52(19 \%)$ & \\
\hline NYHA II & $40 / 73(55 \%)$ & $10 / 21(48 \%)$ & $30 / 52(58 \%)$ & \\
\hline NYHA III & $18 / 73(25 \%)$ & $6 / 21(28 \%)$ & $12 / 52(23 \%)$ & \\
\hline NYHA IV & $0 / 73(0 \%)$ & $0 / 21(0 \%)$ & $0 / 52(0 \%)$ & \\
\hline Weight $[\mathrm{kg}]$ & $81 \pm 19$ & $85 \pm 21$ & $80 \pm 18$ & 0.32 \\
\hline Systolic BP [mmHg] & $118 \pm 18$ & $118 \pm 18$ & $118 \pm 18$ & 0.955 \\
\hline NT-proBNP $[\mathrm{pg} / \mathrm{mL}]$ & $1462 \pm 1964$ & $2015 \pm 2186$ & $1256 \pm 1856$ & 0.152 \\
\hline \multicolumn{5}{|l|}{ Echocardiographic parameters } \\
\hline LVEF [\%] & $38 \pm 10$ & $38 \pm 11$ & $37 \pm 9$ & 0.78 \\
\hline EDVI $\left[\mathrm{mL} / \mathrm{m}^{2}\right]$ & $88 \pm 38$ & $85 \pm 39$ & $89 \pm 37$ & 0.697 \\
\hline IVMD [ms] & $17.3 \pm 28.0$ & $12.9 \pm 20.6$ & $18.8 \pm 30.0$ & 0.41 \\
\hline TDI septal to lateral [ms] & $41.7 \pm 44.7$ & $15.5 \pm 51.2$ & $48.3 \pm 40.8$ & $0.013^{*}$ \\
\hline TDI anteroseptal to inferolateral [ms] & $38.2 \pm 48.4$ & $18.8 \pm 48.8$ & $42.8 \pm 47.7$ & 0.108 \\
\hline \multicolumn{5}{|l|}{ ECG and device parameters } \\
\hline Biventricular pacing [\%] & $95.6 \pm 9.6$ & $93.8 \pm 13.0$ & $96.3 \pm 8.0$ & 0.304 \\
\hline Bundle branch block* & & & & 0.070 \\
\hline LBBB & $54 / 65(83 \%)$ & $7 / 11(64 \%)$ & $47 / 54(87 \%)$ & \\
\hline RBBB & $5 / 65(8 \%)$ & $1 / 11(9 \%)$ & $4 / 54(7 \%)$ & \\
\hline IVCD & $6 / 65(9 \%)$ & $3 / 11(27 \%)$ & $3 / 54(6 \%)$ & \\
\hline Complete AVB & $15 / 80(19 \%)$ & $11 / 22(50 \%)$ & $4 / 58(7 \%)$ & $<0.001 *$ \\
\hline QRS width [ms] & $150 \pm 28$ & $142 \pm 25$ & $152 \pm 28$ & 0.264 \\
\hline $\mathrm{PO}$ interval $[\mathrm{ms}]^{* *}$ & $184 \pm 28$ & $172 \pm 40$ & $185 \pm 27$ & 0.38 \\
\hline Sensed AV interval [ms] & $112 \pm 20$ & $122 \pm 22$ & $110 \pm 19$ & 0.156 \\
\hline Paced AV interval [ms] & $136 \pm 23$ & $146 \pm 31$ & $135 \pm 22$ & 0.223 \\
\hline $\mathrm{VV}[\mathrm{ms}]$ & $10 \pm 17$ & $11 \pm 21$ & $9 \pm 16$ & 0.78 \\
\hline VV changed & $16 / 81(20 \%)$ & $2 / 22(9 \%)$ & $14 / 59(24 \%)$ & 0.141 \\
\hline
\end{tabular}

Continuous variables are presented as mean \pm standard deviation; categorical variables are presented as proportions.

*Complete AVB excluded. **Patients with complete AVB and patients with atrial fibrillation excluded

ACEI/ARB - angiotensin-converting-enzyme inhibitor/angiotensin receptor blocker; AV — atrioventricular; AVB - atrioventricular block;

BP — blood pressure; CRT — cardiac resynchronization therapy; ECG - electrocardiogram; EDVI - end-diastolic volume index; IVCD — intraventricular conduction delay; IVMD - interventricular mechanical delay; LBBB — left bundle branch block; LVEF — left ventricular ejection fraction; NYHA - New York Heart Association; NT-proBNP - N-terminal pro-B-type natriuretic peptide; RBBB - right bundle branch block; TDI - tissue Doppler imaging; VV — interventricular delay 


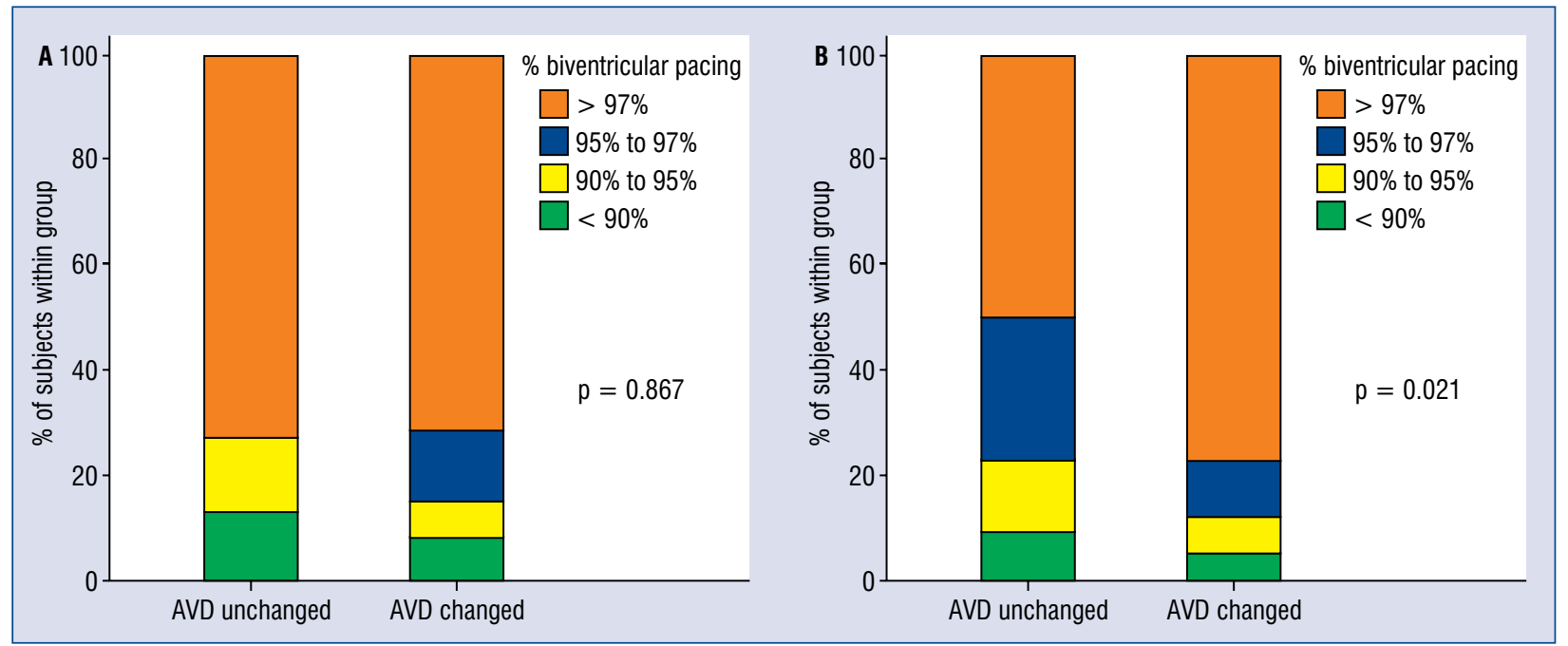

Figure 2. Distribution of biventricular pacing proportions before and after atrioventricular delay (AVD) optimization. Comparison of the intervention (AVD changed) and control (AVD unchanged) group; A. Assessment at baseline; B. Assessment at follow-up. Mann-Whitney U tests.
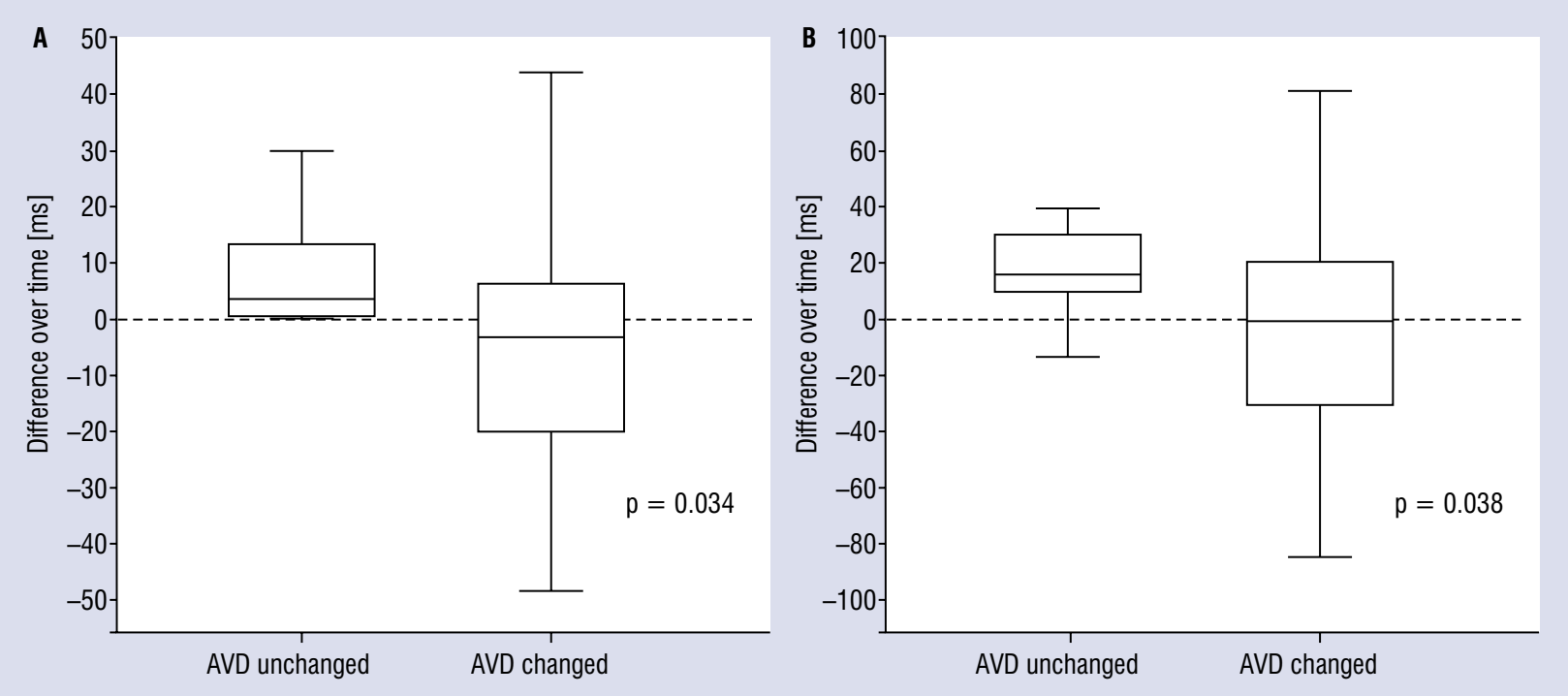

Figure 3. Interventricular mechanical and septal-to-lateral delay in the intervention (atrioventricular delay [AVD] changed) and control (AVD unchanged) group; A. Interventricular mechanical delay; B. Septal to lateral delay. Box plots indicate interquartile ranges, whiskers indicate minima and maxima. Mann-Whitney $U$ tests.

both groups at baseline (Fig. 4C), decreased in the intervention group while it increased in the control group resulting in a significant difference at follow-up (Fig. 4D).

\section{Discussion}

This retrospective is a single-center cohort study in a real-world setting. AVD optimization was associated with an improvement of biventricular pacing, inter- and intraventricular synchronicity, as well as a reduction in mitral regurgitation along with a trend towards reduced end-diastolic $\mathrm{LV}$ volumes.

These results corroborate previous findings from several smaller studies with shorter follow-up [12-14]. However, the role of regular evaluation and adjustment of the atrioventricular interval in 


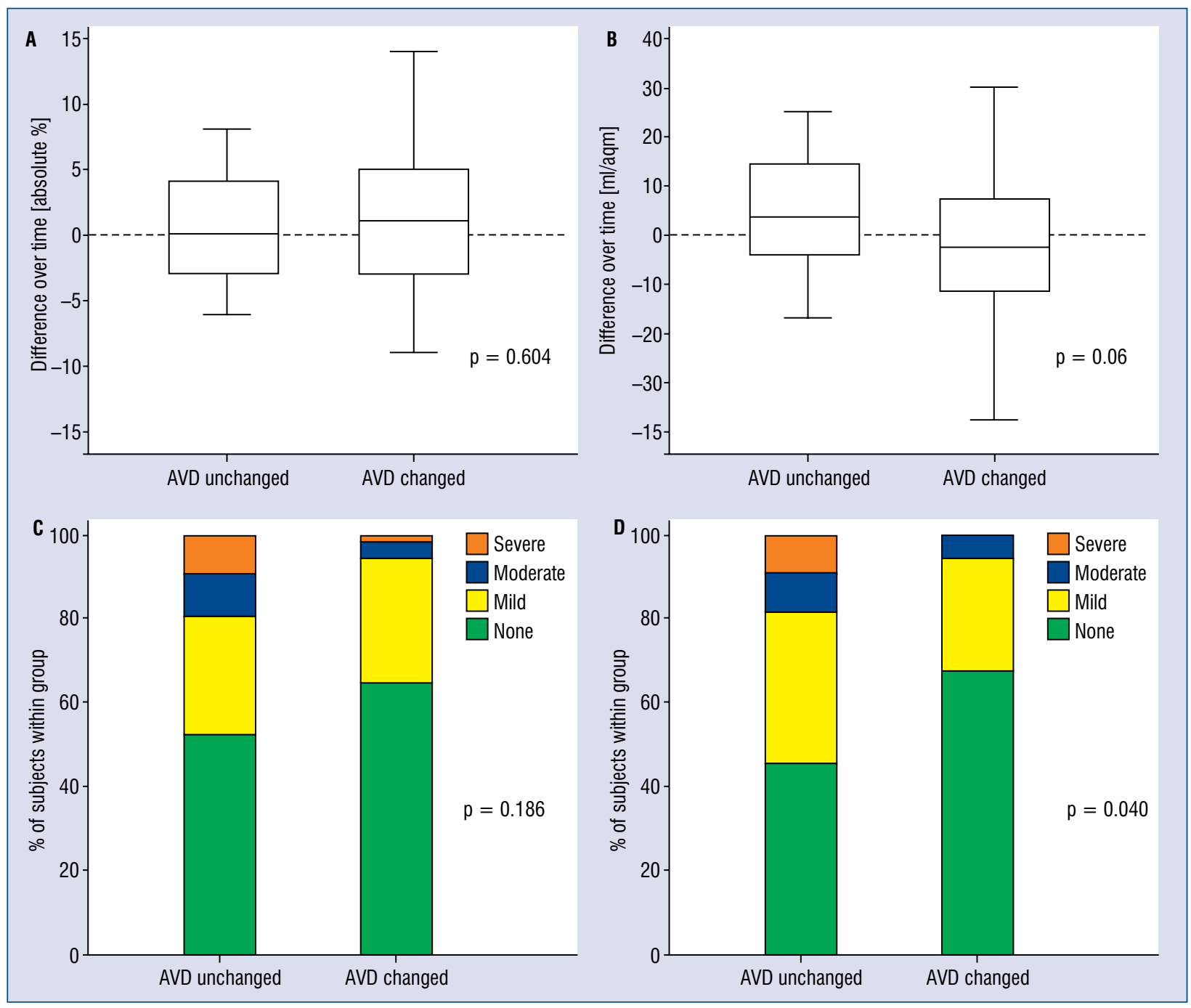

Figure 4. Reverse remodeling upon adapting atrioventricular delays (AVD); $\mathbf{A}, \mathbf{B}$. Change in left ventricular ejection fraction (A) and left ventricular end-diastolic volume index (B), respectively, in the intervention (AVD changed) and control group (AVD unchanged) over time. Box plots indicate interquartile ranges, whiskers indicate minima and maxima; C. Mitral regurgitation at baseline; D. Mitral regurgitation at follow-up. Mann-Whitney $U$ tests.

patients with CRT and the method of AVD optimization remain a matter of debate [20]. In contrast to other studies, the prospective, randomized, controlled SMART-AV trial showed no benefit of general AVD optimization as opposed to a fixed AVD of $120 \mathrm{~ms}$ with regard to the primary outcome of LV end-systolic volume at 6 months [21]. It was concluded that regular AVD assessment and optimization was not necessary and a fixed interval of $120 \mathrm{~ms}$ would suffice. However, these results may not apply to selected individuals, especially those with a suboptimal response to CRT in combination with suboptimal diastolic ventricular filling. Indeed, Mullens et al. [11] observed suboptimal AVD settings in $45 \%$ of those patients who suffered from persistent advanced HF symptoms and/or adverse remodeling after CRT implantation. Furthermore, a sub-analysis of MADIT-CRT, one of the guideline-defining, large randomized, controlled trials, demonstrated that patients programmed to a short AVD $(<120 \mathrm{~ms})$ had a reduced risk of $\mathrm{HF}$ or death over the 3 years following CRT implantation compared to those patients with an AVD $>120 \mathrm{~ms}$, further indicating a role of AVD settings on long-term outcome in selected CRT patients [22]. Finally, a post-hoc analysis of the CLEAR study demonstrated an improved outcome for the composite endpoint of all-cause mortality, $\mathrm{HF}$ hospitalization, NYHA class and quality of life with regular, systematic AVD optimization as opposed 
Table 2. Parameters in patients with changed atrioventricular delay and patients with unchanged atrioventricular delay at follow-up visit.

\begin{tabular}{|c|c|c|c|}
\hline & Control group $(n=22)$ & Intervention group ( $n=59$ ) & $\mathbf{P}$ \\
\hline \multicolumn{4}{|l|}{ Clinical parameters } \\
\hline NYHA class: & & & 0.745 \\
\hline NYHA I & $7 / 21(33 \%)$ & $11 / 54(20 \%)$ & \\
\hline NYHA II & $9 / 21(43 \%)$ & $34 / 54(63 \%)$ & \\
\hline NYHA III & $4 / 21(19 \%)$ & $9 / 54(17 \%)$ & \\
\hline NYHA IV & $1 / 21(5 \%)$ & $0 / 54(0 \%)$ & \\
\hline Weight $[\mathrm{kg}]$ & $83 \pm 24$ & $81 \pm 19$ & 0.603 \\
\hline Systolic BP [mmHg] & $115 \pm 17$ & $120 \pm 16$ & 0.331 \\
\hline NT-proBNP [pg/mL] & $1674 \pm 1446$ & $1092 \pm 1602$ & 0.169 \\
\hline \multicolumn{4}{|l|}{ Echocardiographic parameters } \\
\hline LVEF [\%] & $39 \pm 12$ & $39 \pm 10$ & 0.903 \\
\hline EDVI $\left[\mathrm{mL} / \mathrm{m}^{2}\right]$ & $90 \pm 43$ & $87 \pm 38$ & 0.794 \\
\hline IVMD [ms] & $16.0 \pm 20.6$ & $12.8 \pm 22.3$ & 0.553 \\
\hline TDI septal to lateral [ms] & $31.9 \pm 44.5$ & $47.6 \pm 46.2$ & 0.184 \\
\hline TDI anteroseptal to inferolateral [ms] & $36.2+/-52.3$ & $44.1 \pm 42.5$ & 0.515 \\
\hline \multicolumn{4}{|c|}{ Electrocardiography and device parameters } \\
\hline Biventricular pacing [\%] & $95.3 \pm 6.0$ & $97.5 \pm 4.0$ & $0.034^{*}$ \\
\hline \multicolumn{4}{|l|}{ Bundle branch block* } \\
\hline LBBB & $7 / 11(64 \%)$ & $46 / 54(85 \%)$ & 0.075 \\
\hline RBBB & $1 / 11(9 \%)$ & $5 / 54(9 \%)$ & \\
\hline IVCD & $3 / 11(27 \%)$ & $3 / 54(6 \%)$ & \\
\hline Complete AVB & $11 / 22(50 \%)$ & $4 / 58(7 \%)$ & $<0.001^{*}$ \\
\hline QRS width [ms] & $141 \pm 31$ & $147 \pm 23$ & 0.47 \\
\hline$P Q$ interval $[\mathrm{ms}]^{* *}$ & $189 \pm 40$ & $195 \pm 41$ & 0.92 \\
\hline Sensed AV interval [ms] & $121.8 \pm 20.4$ & $96.4 \pm 28.2$ & $0.006^{*}$ \\
\hline Paced AV interval [ms] & $144.6 \pm 29.8$ & $130.7 \pm 30.5$ & 0.17 \\
\hline $\mathrm{VV}[\mathrm{ms}]$ & $7.6 \pm 15.4$ & $15.7 \pm 22.1$ & 0.144 \\
\hline
\end{tabular}

Continuous variables are presented as mean \pm standard deviation; categorical variables are presented as proportions.

*Complete AVB excluded. **Patients with complete AVB and patients with atrial fibrillation excluded

AV - atrioventricular; AVB — atrioventricular block; BP — blood pressure; CRT — cardiac resynchronization therapy; EDVI - end-diastolic

volume index; IVCD - intraventricular conduction delay; IVMD - interventricular mechanical delay; LBBB - left bundle branch block;

LVEF - left ventricular ejection fraction; NYHA - New York Heart Association; NT-proBNP - N-terminal pro-B-type natriuretic peptide;

RBBB — right bundle branch block; TDI — tissue Doppler imaging; VV — interventricular delay

to "non-systematic" optimization, irrespective of the optimization method applied [23].

Although an assessment of the effect of CRT optimization on morbidity and mortality was beyond the scope of this real-world study, the data support a potential role for CRT optimization with regard to long-term outcome. The present results further underline the importance of the evaluation and adjustment of device settings, given that a substantial part of CRT patients presented with inadequate atrioventricular intervals at baseline.

A high percentage of biventricular pacing is associated with an improved outcome in CRT patients [24]. Koplan et al. [25] demonstrated that the greatest benefit in reduction of HF hospitalization and all-cause mortality was achieved with a biventricular pacing above $92 \%$. The rationale for an even higher proportion of biventricular pacing was provided by Hayes et al. [26] in a cohort of over 30,000 patients, where mortality was found to be inversely related with the percentage of biventricular pacing. Since a reduced percentage of biventricular pacing is among the main reasons for suboptimal response to CRT [11], these data imply that regular assessments and efforts to increase biventricular pacing are central. However, there 
are no data assessing this hypothesis prospectively, and whether certain interventions to improve biventricular pacing such as antiarrhythmic therapy in patients with $\mathrm{AF}$ truly impact hard clinical outcomes remains elusive.

Loss of biventricular pacing can occur as a result of a long AVD due to intrinsic atrioventricular conduction. In such patients, shortening of AVD may increase the degree of biventricular pacing [24-26]. In the present study, patients in whom the AVD was changed (mostly shortened) during CRT optimization had a higher percentage of biventricular pacing at follow-up. The favorable development of hemodynamic parameters in the intervention group may well be a consequence of the higher biventricular pacing proportion in these patients. As adaptation of AVD in order to prevent intrinsic conduction can be performed on the basis of QRS morphology on 12-lead ECG, this raises the question, if echocardiographic assessment during AVD optimization is necessary. It is however important to note that ensuring constant biventricular pacing based on 12-lead ECG alone may lead to programming excessively short AVDs in order to prevent QRS fusion. In this context, echocardiographic monitoring of mitral inflow is crucial in order to avoid impaired left ventricular filling. Herein, echocardiography was therefore regarded as an essential component in the process of AVD optimization.

Taken together, the present findings support the role of systematic AVD optimization to achieve the highest possible percentage of biventricular stimulation and improve hemodynamic parameters. The absolute effect of this improvement, however, was small and it remains to be determined whether this will translate into a reduction in morbidity and mortality.

\section{Limitations of the study}

This study has to be interpreted in light of several limitations, most of which are inherent to any "real-world" registry study. All patients analyzed were recruited at a single center, which may introduce a selection and/or referral bias, and may therefore not reflect the situation in other healthcare facilities.

Furthermore, the control group included 12 patients with AF. In CRT-patients AF can lead to loss of biventricular pacing secondary to high ventricular rates. Importantly, several studies have shown similar benefit of CRT in patients with $\mathrm{AF}$ and those in sinus rhythm [27-30]. However, more recent evidence points to a worse prognosis of CRT in the context of $\mathrm{AF}[31,32]$. This is primarily due to high ventricular rates and consecutive electrical fusion or loss of biventricular pacing, highlighting the importance of adequate rate control [33]. This was evident in the CERTIFY registry by Gasparini et al. [34], in which CRT-patients in sinus rhythm were compared to CRT-patients with AF either after atrioventricular junction ablation (AVJA) or without AVJA [34]. After a median follow-up of 37 months, mortality was similar between AF patients after AVJA and patients in sinus rhythm, while $\mathrm{AF}$ patients on medical rate control alone had a worse outcome compared to both patients in sinus rhythm and patients with AF and AJVA. This implies that patients with AF and complete atrioventricular block derive equivalent benefit from CRT as do patients in sinus rhythm [33]. Out of the 12 patients with $\mathrm{AF}$ in the present cohort, 6 patients had intact intrinsic conduction. Upon exploratory exclusion of these patients, the difference in biventricular pacing between the intervention group and the control group remained significantly different. It can therefore be assumed that the difference in biventricular pacing proportions at follow-up were not driven by patients in $\mathrm{AF}$.

Since this study ought to reflect real-world data, not all variables are distributed evenly between groups. Importantly, there was a higher proportion of patients with complete atrioventricular block in the control group (50\% vs. $7 \%$ ), an effect due to the fact that in these patients AV optimization is oftentimes not necessary as no fusion with intrinsic conduction can occur. Few data exist on the direct comparison between CRT-patients with left bundle branch block and those with complete atrioventricular block. However, in patients with atrioventricular block and reduced LVEF biventricular pacing has been shown to reduce the risk of mortality and morbidity and lead to better clinical outcomes [35]. In the absence of intrinsic conduction, complete atrioventricular block is associated with higher biventricular pacing proportions. Therefore, if present, confounding, may lead to an underestimation of the difference in biventricular pacing proportions in the context of this study. However, as this study was intended to reflect a real-world setting, the current study refrained from excluding patients from the analyses wherever possible. Of note, QRS-width, which is the primary determinant of response in CRT [7], was evenly distributed among the groups in this real-world cohort.

Finally, and as with every registry study, residual confounding between groups may have contributed to the findings; as such, only asso- 
ciations and no causality may be inferred [36]. This notwithstanding, the data herein does reflect a "real-world" setting of CRT patients, which may contribute important insight into evolving therapy concepts such as CRT optimization in daily practice.

\section{Conclusions}

The present study results imply that AVD optimization may result in an increased biventricular pacing percentage, which has been shown to be associated with better hemodynamic parameters and reduced mortality. Whether these hypotheses hold true, remains to be determined in a well-controlled randomized setting.

\section{Acknowledgments}

We are grateful for the continued support of our highly-motivated echo lab- and pacemaker clinic staff.

Conflict of interest: Jan Steffel received consultant and/or speaker fees from Abbott, Amgen, Astra-Zeneca, Atricure, Bayer, Biosense Webster, Biotronik, Boehringer-Ingelheim, Boston Scientific, Bristol-Myers Squibb, Daiichi Sankyo, Medscape, Medtronic, Merck/MSD, Novartis, Pfizer, Sanofi-Aventis, WebMD, and Zoll. He reports ownership of CorXL. Dr. Steffel has received grant support through his institution from Abbott, Bayer Healthcare, Biosense Webster, Biotronik, Boston Scientific, Daiichi Sankyo, and Medtronic. Frank Ruschitzka reports no personal fees; all payments and research grants were paid directly to the University of Zurich. Andreas Flammer received consultant and/or speaker fees from Abbot, Alnylam, Bayer Healthcare, Bristol Myers Squibb, Fresenius, Imedos, Mepha, Novartis, Orion Pharma, Pfizer, Roche and Vifor. Grant support through his institution form Bayer healthcare and Novartis. Stephan Winnik has received educational grants and/or consultant and/or speaker fees from Bayer Healthcare, Daiichi Sankyo, Abbott, and Boehringer-Ingelheim. Grant support through his institution from Abbott.

\section{References}

1. Singh JP, Gras D. Biventricular pacing: current trends and future strategies. Eur Heart J. 2012; 33(3): 305-313, doi: 10.1093/eurheartj/ehr366, indexed in Pubmed: 21951629.

2. Hunt SA, Abraham WT, Chin MH, et al. 2009 focused update incorporated into the ACC/AHA 2005 Guidelines for the Diagnosis and Management of Heart Failure in Adults: a report of the American College of Cardiology Foundation/American Heart Association Task Force on Practice Guidelines: developed in collaboration with the International Society for Heart and Lung Transplantation. Circulation. 2009; 119(14): e391-e479, doi: 10.1161/CIRCULATIONAHA.109.192065, indexed in Pubmed: 19324966.

3. Ponikowski P, Voors AA, Anker SD, et al. 2016 ESC Guidelines for the diagnosis and treatment of acute and chronic heart failure: The Task Force for the diagnosis and treatment of acute and chronic heart failure of the European Society of Cardiology (ESC) Developed with the special contribution of the Heart Failure Association (HFA) of the ESC. Eur Heart J. 2016; 37(27): 2129-2200, doi: 10.1093/eurheartj/ehw128, indexed in Pubmed: 27206819.

4. Cleland JGF, Daubert JC, Erdmann E, et al. Cardiac Resynchronization-Heart Failure (CARE-HF) Study Investigators. The effect of cardiac resynchronization on morbidity and mortality in heart failure. N Engl J Med. 2005; 352(15): 1539-1549, doi: 10.1056/ NEJMoa050496, indexed in Pubmed: 15753115.

5. Abraham WT, Fisher WG, Smith AL, et al. MIRACLE Study Group. Multicenter InSync Randomized Clinical Evaluation. Cardiac resynchronization in chronic heart failure. $\mathrm{N}$ Engl J Med. 2002; 346(24): 1845-1853, doi: 10.1056/NEJMoa013168, indexed in Pubmed: 12063368.

6. Bristow M, Saxon L, Boehmer J, et al. Cardiac-Resynchronization Therapy with or without an Implantable Defibrillator in Advanced Chronic Heart Failure. New Engl J Med. 2004; 350(21): 2140-2150, doi: 10.1056/nejmoa032423.

7. Ruschitzka F, Abraham WT, Singh JP, et al. Cardiac-resynchronization therapy in heart failure with a narrow QRS complex. N Engl J Med. 2013; 369(15): 1395-1405, doi: 10.1056/NEJMoa1306687, indexed in Pubmed: 23998714.

8. Moss AJ, Hall WJ, Cannom DS, et al. Cardiac-resynchronization therapy for the prevention of heart-failure events. N Engl J Med. 2009; 361(14): 1329-1338, doi: 10.1056/NEJMoa0906431, indexed in Pubmed: 19723701.

9. Bax JJ, Gorcsan J. Echocardiography and noninvasive imaging in cardiac resynchronization therapy: results of the PROSPECT (Predictors of Response to Cardiac Resynchronization Therapy) study in perspective. J Am Coll Cardiol. 2009; 53(21): 1933-1943, doi: 10.1016/j.jacc.2008.11.061, indexed in Pubmed: 19460606.

10. Houthuizen P, Bracke FA, van Gelder BM. Atrioventricular and interventricular delay optimization in cardiac resynchronization therapy: physiological principles and overview of available methods. Heart Fail Rev. 2011; 16(3): 263-276, doi: 10.1007/s10741010-9215-1, indexed in Pubmed: 21431901.

11. Mullens W, Grimm RA, Verga T, et al. Insights from a cardiac resynchronization optimization clinic as part of a heart failure disease management program. J Am Coll Cardiol. 2009; 53(9): 765-773, doi: 10.1016/j.jacc.2008.11.024, indexed in Pubmed: 19245967.

12. Sawhney NS, Waggoner AD, Garhwal S, et al. Randomized prospective trial of atrioventricular delay programming for cardiac resynchronization therapy. Heart Rhythm. 2004; 1(5): 562-567, doi: 10.1016/j.hrthm.2004.07.006, indexed in Pubmed: 15851220.

13. Morales MA, Startari U, Panchetti L, et al. Atrioventricular delay optimization by doppler-derived left ventricular $\mathrm{dP} / \mathrm{dt}$ improves 6-month outcome of resynchronized patients. Pacing Clin Electrophysiol. 2006; 29(6): 564-568, doi: 10.1111/j.15408159.2006.00402.x, indexed in Pubmed: 16784420.

14. Hardt SE, Yazdi SH, Bauer A, et al. Immediate and chronic effects of AV-delay optimization in patients with cardiac resynchronization therapy. Int J Cardiol. 2007; 115(3): 318-325, doi: 10.1016/j.ijcard.2006.03.015, indexed in Pubmed: 16891011. 
15. Brugada J, Delnoy PP, Brachmann J, et al. Contractility sensorguided optimization of cardiac resynchronization therapy: results from the RESPOND-CRT trial. Eur Heart J. 2017; 38(10): 730-738, doi: 10.1093/eurheartj/ehw526, indexed in Pubmed: 27941020.

16. Steffel J, Rempel H, Breitenstein A, et al. Comprehensive cardiac resynchronization therapy optimization in the real world. Cardiol J. 2014; 21(3): 316-324, doi: 10.5603/CJ.a2013.0123, indexed in Pubmed: 23990194.

17. Brignole M, Auricchio A, Baron-Esquivias G, et al. ESC Committee for Practice Guidelines (CPG), Document Reviewers. 2013 ESC Guidelines on cardiac pacing and cardiac resynchronization therapy: the Task Force on cardiac pacing and resynchronization therapy of the European Society of Cardiology (ESC). Developed in collaboration with the European Heart Rhythm Association (EHRA). Eur Heart J. 2013; 34(29): 2281-2329, doi: 10.1093/ eurheartj/eht150, indexed in Pubmed: 23801822.

18. Barold SS, Ilercil A, Herweg B. Echocardiographic optimization of the atrioventricular and interventricular intervals during cardiac resynchronization. Europace. 2008; 10 Suppl 3: iii88-iii95, doi: 10.1093/europace/eun220, indexed in Pubmed: 18955406.

19. Gorcsan J, Abraham T, Agler DA, et al. Echocardiography for cardiac resynchronization therapy: recommendations for performance and reporting--a report from the American Society of Echocardiography Dyssynchrony Writing Group endorsed by the Heart Rhythm Society. J Am Soc Echocardiogr. 2008; 21(3): 191-213, doi: 10.1016/j.echo.2008.01.003, indexed in Pubmed: 18314047.

20. Bertini M, Delgado V, Bax JJ, et al. Why, how and when do we need to optimize the setting of cardiac resynchronization therapy? Europace. 2009; 11 Suppl 5: v46-v57, doi: 10.1093/europace/ eup275, indexed in Pubmed: 19861391.

21. Ellenbogen KA, Gold MR, Meyer TE, et al. Primary results from the SmartDelay determined AV optimization: a comparison to other AV delay methods used in cardiac resynchronization therapy (SMART-AV) trial: a randomized trial comparing empirical, echocardiography-guided, and algorithmic atrioventricular delay programming in cardiac resynchronization therapy. Circulation. 2010; 122(25): 2660-2668, doi: 10.1161/CIRCULATIONAHA.110.992552, indexed in Pubmed: 21098426.

22. Brenyo A, Kutyifa V, Moss AJ, et al. Atrioventricular delay programming and the benefit of cardiac resynchronization therapy in MADIT-CRT. Heart Rhythm. 2013; 10(8): 1136-1143, doi: 10.1016/j.hrthm.2013.04.013, indexed in Pubmed: 23712031.

23. Delnoy PP, Ritter P, Naegele H, et al. Association between frequent cardiac resynchronization therapy optimization and longterm clinical response: a post hoc analysis of the Clinical Evaluation on Advanced Resynchronization (CLEAR) pilot study. Europace. 2013; 15(8): 1174-1181, doi: 10.1093/europace/eut034, indexed in Pubmed: 23493410.

24. Gasparini M, Galimberti P, Ceriotti C. The importance of increased percentage of biventricular pacing to improve clinical outcomes in patients receiving cardiac resynchronization therapy. Curr Opin Cardiol. 2013; 28(1): 50-54, doi: 10.1097/ HCO.0b013e32835b0b17, indexed in Pubmed: 23196776.
25. Koplan BA, Kaplan AJ, Weiner S, et al. Heart failure decompensation and all-cause mortality in relation to percent biventricular pacing in patients with heart failure: is a goal of $100 \%$ biventricular pacing necessary? J Am Coll Cardiol. 2009; 53(4): 355-360, doi: 10.1016/j.jacc.2008.09.043, indexed in Pubmed: 19161886.

26. Hayes DL, Boehmer JP, Day JD, et al. Cardiac resynchronization therapy and the relationship of percent biventricular pacing to symptoms and survival. Heart Rhythm. 2011; 8(9): 1469-1475, doi: 10.1016/j.hrthm.2011.04.015, indexed in Pubmed: 21699828.

27. Upadhyay GA, Choudhry NK, Auricchio A, et al. Cardiac resynchronization in patients with atrial fibrillation: a meta-analysis of prospective cohort studies. J Am Coll Cardiol. 2008; 52(15): 1239-1246, doi: 10.1016/j.jacc.2008.06.043, indexed in Pubmed: 18926327.

28. Khadjooi K, Foley PW, Chalil S, et al. Long-term effects of cardiac resynchronisation therapy in patients with atrial fibrillation. Heart. 2008; 94(7): 879-883, doi: 10.1136/hrt.2007.129429, indexed in Pubmed: 18208826.

29. Molhoek SG, Bax JJ, Bleeker GB, et al. Comparison of response to cardiac resynchronization therapy in patients with sinus rhythm versus chronic atrial fibrillation. Am J Cardiol. 2004; 94(12): 1506-1509, doi: 10.1016/j.amjcard.2004.08.028, indexed in Pubmed: 15589005.

30. Delnoy PP, Ottervanger JP, Luttikhuis HO, et al. Comparison of usefulness of cardiac resynchronization therapy in patients with atrial fibrillation and heart failure versus patients with sinus rhythm and heart failure. Am J Cardiol. 2007; 99(9): 1252-1257, doi: 10.1016/j.amjcard.2006.12.040, indexed in Pubmed: 17478153.

31. Boriani G, Gasparini M, Landolina M, et al. Incidence and clinical relevance of uncontrolled ventricular rate during atrial fibrillation in heart failure patients treated with cardiac resynchronization therapy. Eur J Heart Fail. 2011; 13(8): 868-876, doi: 10.1093/ eurjhf/hfr046, indexed in Pubmed: 21558331.

32. Santini M, Gasparini M, Landolina M, et al. Device-detected atrial tachyarrhythmias predict adverse outcome in real-world patients with implantable biventricular defibrillators. J Am Coll Cardiol. 2011; 57(2): 167-172, doi: 10.1016/j.jacc.2010.08.624, indexed in Pubmed: 21211688.

33. Barold SS, Herweg B. Cardiac resynchronization in patients with atrial fibrillation. J Atrial Fibrillation. 2015; 8(4): 1383.

34. Gasparini M, Leclercq C, Lunati M, et al. Cardiac resynchronization therapy in patients with atrial fibrillation: the CERTIFY study (Cardiac Resynchronization Therapy in Atrial Fibrillation Patients Multinational Registry). JACC Heart Fail. 2013; 1(6): 500-507, doi: 10.1016/j.jchf.2013.06.003, indexed in Pubmed: 24622002.

35. Curtis AB, Worley SJ, Chung ES, et al. Improvement in clinical outcomes with biventricular versus right ventricular pacing: the BLOCK HF study. J Am Coll Cardiol. 2016; 67(18): 2148-2157, doi: 10.1016/j.jacc.2016.02.051, indexed in Pubmed: 27151347.

36. Fanaroff AC, Steffel J, Alexander JH, et al. Stroke prevention in atrial fibrillation: re-defining ,real-world data' within the broader data universe. Eur Heart J. 2018; 39(32): 2932-2941, doi: 10.1093/eurheartj/ehy236, indexed in Pubmed: 29688403. 